Mitteilungen der Verbände

Verhaltenstherapie 1996;6:57-67

\title{
Arbeitsgemeinschaft für
}

Verhaltensmodifîkation-Schweiz (AVM-CH)

Kombinierte theoretisch-praktische Kurse für das Sommersemester 1996

(SS 96)

Verhaltenstherapeutische Differentialdiagnostik und differentielle Therapie depressiver Störungen (Prof. Dr. med. Michael Linden, Dipl.-Psych.,

Psychiatr. Klinik der Freien Universität Berlin)

Menschenbild und Ethik in der VT (insb. Therapeut-Patient-Beziehung) (Dr.

phil. Hans Lieb, Dipl.-Psych., Praxisgemeinschaft Edenkoben)

Verhaltenstherapeutische Lernfälle

(Dipl.- Psych. Norbert Kienzle, Heckscher Klinik München)

Standardmethoden der Verhaltenstherapie und Indikationsfragen II -

Kognitive Verfahren (Frau Dr. phil. U. Willutzki, Dipl.- Psych. Universität

Bochum)

Verhaltenstherapeutische Gruppentherapie

(Dr. phil. Jörg Petry, Dipl.-Psych. Psychosomatische Fachklinik

Münchwies)

Kosten (2tägiger Kurs): $\quad$ AVM-CH Mitglieder mit

Weiterbildungsvertrag

AVM-CH Mitglieder andere 
16717.3.96 (Sa, So)

12./13.4.96(Fr.Sa)

12./13.4.964

$1 / 2.6 .96(\mathrm{Sa}, \mathrm{So})$

29./30.6.96(Sa,So)

230-sFr. 240- sFr. 290- sFr. 
Falls auf der Anmeldebestätigung keine anderen Angaben gemacht werden, beginnen alle Kurse am ersten

Tag um 9.00 Uhr. Ein Kurstag umfaßt 8 Ausbildungsstunden (à 45 Min.).

Die Kurse 1, 2 , 3 finden im Hörsal der Alien Klinik der UPD Bern, Bolligenstraße 111, Ostermundigen statt.

Die Kurse 4, 5 werden an der Psychiatrischen Klinik Hard, 8424 Embrach durchgeführt.

Die Teilnehmerzahl der Kurse ist limitiert. AVM-CH-Mitglieder erhalten Seminarplätze in erster Präferenz.

Die Kurse sind Bestandteil des Weiterbildungscurriculums der AVM-CH zum Verhaltenstherpeuten/zur

Verhaltenstherapeutin.

Hinweis Voraussichtlich beginnt ab dem Wintersemester 96/97 ein neuer 2- bzw. 4jähriger Weiterbildungs-gang in Verhaltenstherapie. Anfrage bitte an u. g.

Anschrift.

AVM-CH

Frau U. Keppeler

Universitäre Psychiatrische Dienste Bern

Bolligenstraße 111

CH-3872 Ostermundigen

Tel. 031/8292484 
7 (12)

$\propto \operatorname{Lieb}(12 . / 13.4 .96) \propto \operatorname{Petry}(29 . / 30.6 .96)$

$\propto$ Kienzle $(374.5 .96)$

$\propto$ Linden (16/17.3.96) $\propto$ Willutzki (172.6.96)

(1).6.96)

Name Vorname 
Anschrift 
Beruf.

Unterschrift 
Mitteilungen der Verbände 
Mit meiner Unterschrift erkläre ich verbindlich meine Seminarteilnahme. Bei Abmeldungen, die schriftlich mehr als 4 Wochen vor Kursbeginn erfolgen, werden $50 \%$ der Gebühren verrechnet. Bei kürzer vorgenommenen Abmeldungen müssen die vollen Seminarkosten beglichen werden. Falls ein Ersatzteilnehmer von der angemeldeten Person gefunden wird, erfolgt in jedem Fall die Rückerstattung bzw. Nicht-Berechnung der Gebühren.

Anmeldetalon senden an: Frau Lie. phil. D. Heimberg. PUK, Wilhelm-Klein-Str. 27, 4025 Basel (Fax 061/3255513) Informationen zu den Kursen: $061 / 325$

5113 (Frau Heimberg, vormittags)

* Postscheckamt Bern, Postscheckkonto-Nr. 30-9429-8, Kontobezeichnung AVM-CH 
C 1996 S. Karger

GmbH, Freiburg Fax

(0761) 4520714 
Arbeitsgemeinschaft für Verhaltensmodifíkation e.V. (AVM-D)

Im Rahmen eines Projekts «lntegration von Theorie und Praxis» der Arbeitsgruppe Interventionspsycho-logie am Institut für

Psychologie der Universität Würzburg entsteht eine Psychologische Forschungs- und Beratungsstelle. Ihre Aufgabenbereiche sind

Forschung, Aus ${ }^{-}$, Fort- und Weiterbildung in Methoden der Interventionspsychologie und Durchführung psychologischer

Interventionsmaßnahmen. Als Rechtsform ist ein gemeinnütziger Verein vorgesehen. Die Aktivitäten der Psychologischen

Forschungs- und Beratungsstelle, insbesondere die Forschung, erfolgen in Anbindung an die Universität (akademische Leitung: Prof.

Dr. Heiner Ellgring). Anträge auf Bewilligung einzelner Forschungsprojekte werden vorbereitet, Vor-gespräche mit möglichen

Förderinstitutionen sind im Gange. Im Vordergrund stehen derzeit Fragen der psychologischen Intervention bei körperlichen

Erkrankungen, v. a. deren psychischen Folge- und Begleit-erscheinungen.

Zweitens ist vorgesehen, Kenntnisse und Fertigkeiten in empirisch-wissenschaftlichen und überprüften Methoden der

Interventionspsychologie zu vermitteln, z. B. durch Praktika für Studierende der Psychologie, studienbegleitende oder postgraduierte

Weiterbildungsmaßnahmen in Zusammenarbeit mit der Arbeitsgemeinschaft für Verhaltensmodifîkation (AVM) und anderen

verhaltenstherapeutischen Institutionen, sowie auch in Form von Vorträgen und Workshops.

In einem dritten Arbeitsbereich «lnterventionen» (Beratung, Therapie) werden psychologische Interventionsmaßnahmen, basierend auf den Ansätzen der kognitiven Verhaltenstherapie, von Diplom-Psychologen im Rahmen einer postgraduierten Weiterbildung durchgeführt. Die Finanzierung erfolgt weitgehend über das Kostenerstattungsverfahren durch Krankenkassen. Gemeindenahe therapeutische Projekte (z.B. an-geleitete Selbsthilfegruppen) werden angestrebt. Die Arbeitsgemeinschaft für Verhaltensmodifîkation (AVM-D) hat einen Antrag der Psychologischen Forschungs- und Beratungsstelle auf Anschubfinanzierung für die Zeitdauer von 17 Monaten (November 1995 bis März 1997) gewährt. Diese Förderung wird zur Anmietung von Räumen und für die stundenweise Anstellung einer Technischen Assistenz verwendet. Die Organisatoren der Psychologischen Forschungs- und Beratungsstelle wurden durch die Genehmigung des Antrags in ihrer Motivation zur Fortführung des Projekts bestärkt und sprechen der AVM an dieser Stelle noch einmal ihren herzlichen Dank aus.

Heiner Ellgring Machael Macht Ralph Schwarz

AVM-Geschäftsstelle:

Lehrstuhl für klinische Psychologie

c/o Frau Dagmar Frey

Universität Bamberg

Postfachl549

D-96045 Bamberg

Mitteilungen der Verbände 
Verhaltenstherapie 1996;6;57-67 
Bayerische Private Akademie für Psychotherapie GmbH (BAP)

Kinder- und Jugendtherapieausbildung 1996

Das verhaltenstherapeutische Kinder- und Jugendlichentherapie-Curriculum besteht aus:

100 Stunden Theorie und fallzentrierte praktische Ausbildung (6 Wochenendworkshops)

5 eigene Kinder- oder Jugendlichenbehandlungen unter Supervision

durchschnittlich jede 4. Therapiestunde eine Stunde Supervision Ausbildungsleitung: Dr. F. Peterander, Dr. Dr. S. Sulz

Supervisoren u. Dozenten: Dr. H. Dirlich-Wilhelm, Dr. B. Hippler, G. Görlitz, Dr. F Peterander, Dr. Dr. S. Sulz

Gruppentherapie- Ausbildung 1996

Die Tätigkeit als Gruppen-Verhaltenstherapeut erfordert angesichts spezieller Probleme und Behandlungs-bedingungen neben einer qualifizierten Ausbildung zur Behandlung Erwachsener zusätzlich Kenntnisse und Erfahrungen in der Theorie und Praxis der

Gruppenbehandlung. Unsere Gruppentherapieausbildung vermittelt während 7 Wochenendworkshops à 16 Stunden:

Sozialpsychologische Grundlagen

Verhaltenstherapeutische Grundlagen

Gruppen-Diagnostik

Gruppenbehandlung

V Selbsterfahrung in der Gruppe

Teilnehmende Beobachtung und Kotherapie bei Gruppentherapien

Durchführung eigener Gruppenbehandlungen unter Supervision. Ausbildungsleitung: Dr. Dr. S. Sulz, Dr. R. Ullrich de Muynck

Supervisoren u. Dozenten: Dr. C. Brandl, Prof. W. Butollo, G. Görlitz, Dr. B. Hippler, Dr. Dr. S. Sulz, Dr. R. Ullrich, Dr. R. Ullrich de Muynck

Information und Anmeldung: Bayerische Akademie für Psychotherapie Nymphenburger Straße 18580634 München Tel. (089)13

079315

Mitteilungen der Verbände 
Verhaltenstherapie 1996;6;57-67 
Centrum für Integrative Psychotherapie (CIP)

1. 6. Bayerische Verhaltenstherapietagung vom 10. bis 12. Mai 1996: Wie geschieht psychische Heilung? Zentrale Prozesse in der

Therapie-Interaktion

Wissenschaftliche Vorträge

Freitag, den 10. Mai 1996, ab 13.00 Uhr

Dr. Dr. S. SulzHeilung durch Lernen

Prof. W. Butollo Heilung im Spannungsfeld von Handeln und Erfahrung

Dr. G. Röper Heilung durch Entwicklung

Dipl.-Psych. W. Scholz Heilung durch Beziehung

Dr. F. Giesen Heilung durch den Körper

Anschließend Podiumsdiskussion

In diesem Jahr veranstaltet das Centrum für Integrative Psychotherapie CIP erne sechste Bayerische Ver

haltenstherapietagung. Die Veranstaltungen sind auf unterschiedliche Berufsgruppen ausgerichtet und

sollen in möglichst praxisnaher Form Verständnis und Wissen zu diagnostischen und therapeutischen

Themen vermitteln.

!! Inhaltlicher Schwerpunkt des Tagungsangebots ist in diesem Jahr der Prozeß der Heilung, d. h. wie wichtige

Teilaspekte der Heilung in Therapie-Interaktion umgesetzt werden.

Die Vorträge am Freitag behandeln dieses Thema, wobei viel Zeit zur Diskussion eingeräumt wird. Am Samstag und Sonntag kann

ein vertiefender Kurs besucht werden.

Die im Rahmen der Tagung stattfindenden Kurse richten sich überwiegend an Ärzte und Diplompsycho-logen, die sich für

Fortbildung und Weiterbildung interessieren. Dabei können die einzelnen Teilnehmer entscheiden, ob sie die Angebote der

Weiterbildung zur verhaltenstherapeutischen Psychotherapiezusatz-bezeichnung wahrnehmen wollen oder als bereits praktizierende

Psychotherapeuten oder Psychiater ent-sprechende Fortbildungsangebote wählen. Auf die Einbeziehung von Angeboten zur

psychosomatischen Grundversorgung wurde ebenfalls Wert gelegt. Hiermit soil eine Brücke zwischen der somatischen Medizin und der Psychotherapie zum Vorteil der Patienten geschlagen werden.

Wochenendseminare

So/So 11/12. Mai 1996, 9.00-17.15 Uhr

Körperliches Lernen: Heilung durch körperorientiertes Vorgehen in der Verhaltenstherapie

] Dipl.-Psych. Gudrun Görlitz

Körperorientierte Interventionen stellen eine wichtige Ergänzung zu verhaltensorientierten rational-emotiven Methoden dar. Übungen zur Verbesserung der Körperwahrnehmung und des Körperbewußtseins bringen darüber hinaus viel Spaß und Lebendigkeit in die therapeutische Arbeit für Patienten und Therapeuten.

Soziales Lernen: ATP. Assertiveness-Training bei sozialer Angst 
Dr. med. R. Ullrich, Arzt f. Psychiatrie, Neurologie - Psychotherapie-Verhaltenstherapie

Soziales Lernen und soziale Ängste sind die zentralen Größen in der Gestaltung sowohl der klinisch unauf-

fälligen als auch der gestörten Persönlichkeit. Der lerntheoretisch-bedingungsanalytische Zusammenhang

soil hinsichtlich Indikationsstellung und Therapieableitung dargestellt werden. Zentrale therapeutische

Strategien aus dem Selbstsicherheitskurs und dem Kommunikationskurs des ATP werden an praktischen

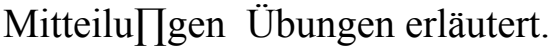

der Verbände

Neurolingustisches Lernen: NLP Sprache, Metaphern, Geschichten

Dr. med. Arnold Torhorst, Arzt f. Psychiatrie, Psychotherapie u. Psychotherapeutische Medizin - Psychoanalyse

NLP ist ein Modell, das Wege und Möglichkeiten aufzeigt, wie die gesunden Eigenkräfte im Menschen ent-deckt, entfaltet und für

Problemlösungen wirksam eingesetzt werden können. Das Modell geht davon aus, daß jeder Mensch sowohl in jeder problematischen

Situation mehr Wahlmöglichkeiten hat, als ihm bewußt zugänglich sind, als auch in seinem eigenen Erfahrungsreichtum alle die

Möglichkeiten finden kann, um die Probleme angenehmer und in angemessener Weise zu bewältigen. 
Verhaltenstherapie 1996;6:57-67 
Motivation zur Veränderung: Aufbau von Therapiemotivation Dipl.-Psych. Dieter Schmelzer $, \mathrm{n}-, \mathrm{rv} \quad * \quad,,, \mathrm{r} . \mathrm{r} \prod$ Vií»rmn 7П einer Therapie motiviert seien, geht die $\operatorname{Tm} \Gamma^{1} / 8 \sigma p \prod$ satz zu der Vorstellung, daß Khenten von vornherein $\mathrm{zu}^{\left.\mathrm{TM}_{>}\right) \mathrm{j}} \mathrm{p}$

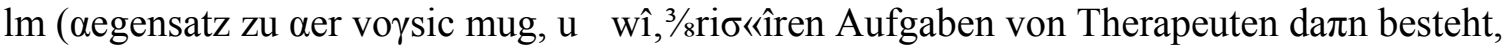

Selbstmanagement-Therapie davon aus, daß eine der wichtigsten Aug

Klienten zu motivieren - und zwar vor allem zu Veränderungen- Alle Mo $\mathrm{i}^{\wedge}{ }^{\wedge}$ tionsstrategien mussen un

jviiemen zu mui $\wedge$ wíirl·Qjrhtioen· andernfalls sind uber kurz oder lang

bedingt die zentralen «Lebensziele» von Khenten berucksichtigen, \&lt; $\chi$

Phänomene zu erwarten, die als «Widerstand» bezeichnet werden.

Erfahrungsorientiertes Lernen als Psychotherapie: ELT

Prof. Dr. Siegfried Höfling

ELT is, em von Butollo u,,d Hõfling 1984 en,»ickeltes T ${ }^{1} / 8$ erapieko»zept. das verhalte,,s, he, apeu, ische. ge-

fp $\ll h o^{1 / 8 e}, a^{1 / 8 \wedge}$

und dadurch zeitökonomische Vorteile bringt.

Fallseminar· Strategie der psychischen Entwicklung und ihre Störungen

D7 ГГ Г Р Phil. Dipl,Psych. SKD Sulz, Arzt für Psychiatrie, Psychotherapy und Psychotherapeutische

Medizin

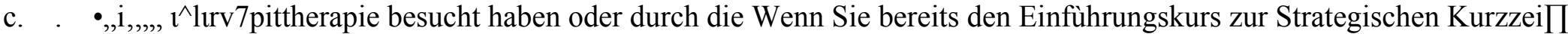

Lektüre der Bücher «Strategische Kurzzeittherapie» begonnen haben, nach diesem Konzept zviabeiten, so

L.ekmre uei Duv.nk.1 ot \& Cvmervisionsseminar wird das Fallverstand-

können Sie in dieses Seminar Ihre Falle mitbrmgen. Im Fall- und Supe Veränderung

nis und die individuelle dysfunktionale Homöostase erarbeitet, der kürzeste Weg zvüeren veränderung

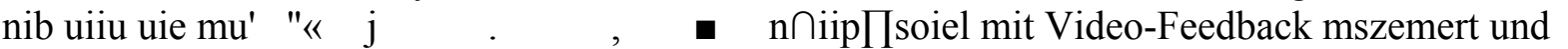

gesucht und das praktische therapeutische Vorgehen im Rollenspiei

reflektiert: Wie zum Wesentlichen gelangen und dabei bleiben?

Lit. S. Sulz: Praxis-Manual zur Strategischen Kurzzeittherapie. ClP-Meüien isw

Das ausführliche Tagungsprogramm erhalten Sie beim CIP-Centrum (siehe unten).

2. Weiterbildungsprogramm: Theorie und Praxis der VT 
Dr Wolfeang Hiller, ltd. Psychologe, Psychosomatische Klinik Roseneck P $\pi$ en

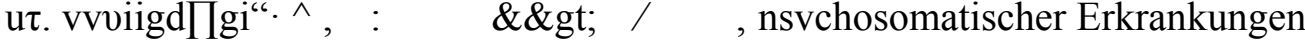
Befunderhebung, Diagnose und Differenüaldiagnose psychischer un $\alpha$ psyc $\pi v^{1 / 80}$

$\mathrm{Sa} / \mathrm{So}, 23 . / 24.3 .96$

Dr Dr S K.D. Sulz, Arzt für Psychiatrie, Psychotherapie und psychotherapeutische Medizin

Strategische Kurzzeittherapie - Wege zur effizienten Psychotherapie Samstag, 20.4. - Dienstag, 23.4.96

Dipl.-Psych. H.-U. Schachtner, Psychotherapeut, München Aussöhnung

Sa/So, Ó./7.7.96

Barbara Y Rakow, Krankenschwester für Psychiatrie, Ingolstadt

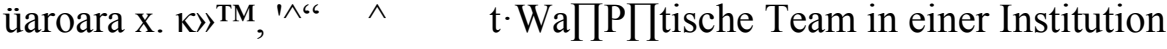

Teamarbeit - alle reden davon, mrgends klappt s? Das therapeutist

Für Mitarbeiter in Psychiatrie und Psychosomatik

$\mathrm{Sa} / \mathrm{So}, 677.7 .96$

Centrum für Integrative Psychotherapie CIP

Nymphenburger Straße 185

80634 München

Tel. (0 89) 1307 9315, Fax (0 89) 132133

Bei Interesse bitte ausßhrlíches Programm anfordern. 
Mitteilungen der Verbände 

Verhaltenstherapie 1996;6;57-67 
Deutscher Fachverband für Verhaltenstherapie (DVT) Ausschuß der Institute im DVT

Die im DVT organisierten Institute trafen sich im Jahr 1995 am 19.1., am 30.6. und am 24.11. Die Diskus-sionen wurden über zukünftige Kooperationen mit den Hochschulen und anderen Verbänden in Verhaltenstherapie sowie über verbandsinterne QualitätsStandards geführt.

Die Institute befürworten eine enge Kooperation mit Instituten an deutschen Universitäten, die die Klini-sche Psychologie repräsentieren, aber auch mit anderen Einrichtungen, die psychotherapeutische Forschung betreiben und insbesondere auch mit universitären Kliniken der Psychiatrie. Bei der Kooperation sollen neue Wege erprobt werden, die eine einseitige Bevormundung verhindern. Wie frühere Umfragen ergeben haben, haben de facto die meisten Institute Mitarbeiter der Universität in ausbildungsrelevanten Gremien. Alle arbeiten mit Dozenten von der Hochschule zusammen.

Alle zwei Jahre sollen die Gebühren der Ausbildungsinstitute erhoben werden und als Mittelwerte und Streuungen den Instituten zur Orientierung bekanntgegeben werden. Eine erste Erhebung wurde 1995 durchgeführt.

In mehreren Schritten wurden die im folgenden abgedruckten Qualitätsstandards erarbeitet und am 24.11.1995 verbindlich verabschiedet.

Tubingen, den 20.12.1995

Prof. Dr. Dirk Zimmer

Sprecher der Ausbildungsinstitute im DVT

Waldhäuserstraße 48

72076 Tubingen

Tel. (07071)610838

Fax (07071) 760243 
Mitteilungen der Verbände 
Qualitätsstandards für Weiterbildungsinstitute im DVT

Die im Deutschen Fachverband für Verhaltenstherapie (DVT) organisierten Institute haben am

24.11.1995 in Köln die folgenden Kriterien als Minimalstandards für DVT-Institute verabschiedet. Sie werden in Zukunft weiterentwickelt und setzen als selbstverständl·ich voraus, daß die Institute die jeweils geltenden Psychotherapierichtlinien beachten, die hier nicht wiederholt werden.

1. Formale Qualitätskriterien

Weiterbildungsvertrag: Weiterbildungsverträge können sich auf die gesamte Weiterbildung erstrecken. Die Kandidaten sind darüber zu informieren, daß die Verträge mit angemessener Kündigungsfrist zum jeweils nächsten Semester/Halbjahr gekündigt werden können.

Schweigepflicht: Für Ausbildungskandidaten, Dozenten, Supervisoren und Selbsterfahrungsleiter gelten die allgemeinen Bestimmungen der Schweigepflicht.

Die Ausbildung hat in festen Ausbildungsgruppen mit einem gemeinsamen geschlossenen

Curriculum für die Theorie-Praxis-Seminare stattzufinden. Die Kursgröße bei Praxis-Seminaren

darf die Zahl 20 nicht überschreiten.

Das Institut hat Sorge zu tragen, daß Ausbildungskandidaten relevante Ausbildungsteile nachholen können, wenn sie diese aus wichtigen Gründen - etwa wegen Krankheit - nicht besuchen konnten.

Ausbildungsinstitute sollten daher regelmäßig, spätestens alle zwei Jahre, einen neuen

Weiterbildungszyklus beginnen.

Ausbildungsinstitute beziehen in ihre Gebührenordnungen die Orientierungswerte ein, die sich aus den alle zwei Jahre durchzuführenden Erhebungen (zu den Gebühren bei DVT-Instituten) und den daraus resultierenden Durchschnittswerten ergeben.

Dozenten und Supervisoren sind vom Institut in einem angemessenen Verhältnis zu den

Teilnehmern zur Verfügung zu stellen (ein Supervisor auf vier Teilnehmer), wobei ein Großteil der Dozenten und Supervisoren vor Ort verfügbar und ansprechbar sein muß. Supervisoren sollten regelmäßig an der Lehre beteiligt werden. 

Verhaltenstherapie 1996;6;57-67 
Das Institut hat eine Ausbildungsambulanz zu führen, die mit der erforderlichen räumlichen, tech-nischen und personellen Ausstattung als eigene Institution der psychotherapeutischen Versorgung er-kennbar ist. Alle dort durchgeführten Behandlungen sind adäquat zu supervidieren. Der zur Beauf-tragung ermächtigte Arzt muß ansprechbar sein und zu regelmäßigen Terminen für Patientengespräche zur Verfügung stehen.

Die Verantwortungsstruktur des Ausbildungsinstitutes ist für Kandidaten transparent zu machen.

Das Institut hat durch geeignete Maßnahmen Sorge zu tragen, daß Anregungen und Rückmeldungen, Wünsche und Kritik der Ausbildungskandidaten regelmäßig erfragt und in die Planungen einbezogen werden.

2. Kandidatenauswahl

Die fachlichen und persönlichen Voraussetzungen für eine psychotherapeutische Ausbildung müssen am Anfang und fortlaufend während der Weiterbildung angesehen werden. Das Institut hat ein geeignetes Procedere für Problemfälle zu entwickeln.

3. Supervision

Das Institut führt eine Liste von Supervisoren, die von der KBV anerkannt sind, und über deren Eignung sich die Leitung des Institutes ein Urteil gebildet hat.

Im Prinzip besteht freie Wahl der Supervisoren. In als notwendig erachteten Fallen kann das Institut Weiterbildungskandidaten gezielt bestimmten Supervisoren zuordnen.

Maximal die Hälfte der Einzel- oder Gruppensupervisionsstunden darf in einem Abhängigkeitsverhält-nis zum Supervisor, etwa dem Vorgesetzten oder Oberarzt im Rahmen einer Anstellung, durchgeführt werden.

4. $\quad$ Selbsterfahrung

In der Regel sollte ein Ausbildungskandidat nicht zur gleichen Zeit bei derselben Person in Supervision und Selbsterfahrung sein, da Supervision und Selbsterfahrung unterschiedliche Rollenbeziehungen implizieren. In einem Fall steht die Verantwortung dem

Patienten gegenüber, beim anderen die persön-liche Entwicklung des angehenden Therapeuten im Vordergrund.

Es darf keine wirtschaftliche oder berufliche Abhängigkeit zum Selbsterfahrungsleiter bestehen.

In der Regel kommt der Selbsterfahrungsleiter als Prüfer nicht in Frage, es sei denn auf Wunsch des Ausbildungskandidaten.

Das Institut hat Sorge zu tragen, daß mit der Selbsterfahrung möglichst früh in der Ausbildung begon-nen wird. Bis zur

Zwischenprüfung sollten mindestens 50\% der Selbsterfahrung abgeleistet werden.

Mitteilungen der Verbände 
Verhaltenstherapie 1996;6:57-67 
Institut für Verhaltenstherapie Berlin e.V. (ГVB)

Fortbildungsveranstaltungen für Diplompsychologen und Ärzte

Sexueller Mißbrauch

Dozentin: Dipl.-Psych. U. Schiller

Termine: 9.3.1996 und 10.3.1996 (10.00-17.00 Uhr)

Die entwicklungspsychologische Perspektive in der Verhaltenstherapie

Dozentin: Dr. phil. G. Röper

Termine: 23.3.1996 (11.00-18.00 Uhr) und 24.3.1996 (10.00-17.00 Uhr)

In-vivo- Therapie

Dozent: Dipl.-Psych. Dr. phil. N. Hoffmann

Termine: 20.4.1996 und 21.4.1996 (10.00-17.00 Uhr)

Die Hörergebühr beträgt für alle Veranstaltungen (je 16 Unterrichtseinheiten) 395,- DM; ermäßigt 315,- DM.

Veranstaltungsort und Anmeldung:

Institut für Verhaltenstherapie e.V, Berlin

Teltower Damm 7

14169 Berlin-Zehlendorf

Tel. 8112044, Mo, Di, Mi, Fr: 9.00-11.00 Uhr, Do: 17.00-19.00 Uhr

Mitteilungen der Verbände 
Verhaltenstherapie 1996;6;57-67 
Tübinger Akademie für Verhaltenstherapie (TAVT)

Für die Teilnehmer an einer Weiterbildung der TAVT aus dem Konstanzer Raum wurde in

Konstanz eine Ambulanz unter ärztlicher Leitung von Dr. Anselm Grusche eingerichtet und von der Kassenärztlichen Bundesvereinigung anerkannt.

Der nächste Ausbildungsgang an der TAVT beginnt wieder im Oktober 1996. Das obligatorische

Auswahl-seminar hierfür findet am 24.-25. Juni in Tubingen statt.

Weitere Blockveranstaltungen, an denen auch Gäste teilnehmen können, sind:

14.-15.4.96 M. Smucker Kognitive und imaginative Verfahren zur Bewältigung

traumatischer Erinnerungen bei Opfern sexueller Gewalt

16.-17.4.96 M. Smucker Behandlung von Borderline-Patienten

25.-27.4.96 D. Zimmer/F.T. Zimmer Einführung in Paartherapie

31.5.-1.6.96 S. Fliegel Behandlung von Panikstörungen und Phobien

9.-10.6.96 F. H. Kanfer Selbstmanagement-Therapie 1

2.-3.7.96 F. H. Kanfer Selbstmanagement-Therapie 2

12.-13.7.96 M. Reimer VT bei Zwangsstörungen

20.-21.9.96 J. Weidhaas/A. v. Pein Funktionelle Störungen

22.-23.9.96 J. Weidhaas Organisatorische und Rechtsfragen ambulanter Psychotherapie im Rahmen freiberuflicher psychotherapeutischer Praxis

17.-19.10.96 FT. Zimmer/D. Zimmer Kognitive Verhaltenstherapie der Depressionen

15.-16.11.96 W. Langosch VT-Strategien zur Streßbewältigung

Nähere ìnformationen und Auskünfte sind über das Sekretariat erhältlich:

TAVT

Waldhäuserstraße 4872076 Tubingen Tel. (07071)610838 Fax (07071) 760243

Verhaltenstherapie 1996:6;57-67 
Mitteilungen der Verbände 
Vereinigung der Kassenpsychotherapeuten 
Mitteilungen der Verbände 
Anfang des Jahres erschien eine Broschüre der Vereinigung zur Situation der Psychologischen Psychotherapie

in der Privaten Krankenversicherung (PKV). Die Broschüre enthält die Ergebnisse einer umfassenden Er-

hebung unter den privaten Versicherern über ihr Erstattungsverhalten bei Psychotherapie, speziell bei psycho-

logischer Psychotherapie. Die Auswertung ergab folgenden Eindruck:

Während ein privat Versicherter sich in der Regel darauf verlassen kann, daß seine Versicherung die Kosten

für ärztliche Behandlung übernimmt, ist die Erstattung psychologischer Psychotherapie dagegen

keine

Selbstverständlichkeit.

Bei den privaten Versicherungen herrscht eine Vielfalt unterschiedlicher

Versicherungsbedingungen. Sie

unterscheiden sich hinsichtlich:

Der Erstattung psychologischer Psychotherapie überhaupt. Einige Versicherungen lehnen es grundsätzlich ab, diese Leistungen zu bezahlen (z. B. HUK Coburg, Inter, LKH, Universa), während die meisten anderen psychologische Psychotherapie zu gleichen Bedingungen erstatten wie Psychotherapie, die von einem Arzt geleistet wird.

Der Vertragssicherheit. D. h.: Während bei der Mehrzahl der Versicherungen der Leistungsbereich Psychologische Psychotherapie Vertragsbestandteil ist, erstatten andere nach Einzelfallprüfung als sogenannte Kulanzleistung. Hier steht es im Ermessen der Versicherung, oft nach Konsultation eines Gutachters, eine Psychotherapie zu befürworten oder abzulehnen.

Der Stellung, die dem Psychologischen Psychotherapeuten eingeräumt wird. Einige

Versicherungen (z. B. Barmenia, Mannheimer, Union) erstatten Psychotherapie ohne vorherige Antragstellung und ohne ärztliche Abklärung, andere wiederum, wie etwa die Niirnberger, DKV, Hallesche Nationale, SAVAG, Victoria, verlangen ärztliche «Überwachung», «Verantwortung» oder «Kontrolle».

-Der Stundenkontingente. Da Psychotherapie anscheinend zu den besonders wenig kalkulierbaren Kostenfaktoren zählt, legen die meisten Versicherungen Höchstgrenzen fest: entweder in der Sitzungs-anzahl pro Jahr, im Gesamthonorar für Psychotherapie pro Jahr oder in der Beschränkung des GOÄ-Multiplikators. 
Der Honorierung ärztlicher im Vergleich zu Psychologischer Psychotherapie. Einige Versicherungen erstatten bis zum 2,3fachen der GOÄ-Sätze, wenn ein Arzt die Psychotherapie durchführt, aber nur das 1, ófache bei psychologischer Psychotherapie (z. B. Alte Oldenburger, Colonia, DBV, Generali).

Des Gutachterverfahrens. Einige Versicherungen wenden kein Gutachterverfahren an, während andere jede Therapie, auch Kurzzeittherapie, begutachten lassen.

Qualifikationsvoraussetzungen des Therapeuten. Einige Versicherungen verlangen eine Ausbildung an einem KBV-anerkannten Institut in einem der Richtlinienverfahren (Psychoanalyse,

Verhaltenstherapie oder tiefenpsychologisch fundierte Psychotherapie) bzw. Kassenzulassung. Für die Mehrzahl der Versicherungen ist das Diplom in Psychologie ausreichend.

Der Qualifikation des «delegierenden» bzw. verordnenden Arztes. Die Orientierung mancher Versicherungen an den Psychotherapierichtlinien erfordert einen vorausgehenden Besuch des Patienten beim ärztlichen Psychotherapeuten, während bei den meisten anderen Gesellschaften eine (haus-)ärztliche Ver-ordnung ausreicht.

Einige Versicherungen lehnen sich an das vom Bundesausschuß der Ärzte und Krankenkassen für den Bereich der Gesetzlichen Krankenversicherungen in den Psychotherapierichtlinien festgelegte Delegations-verfahren an. Dies bedeutet, die Therapie beim Psychologischen Psychotherapeuten soil unter der Gesamt-verantwortung eines ärztlichen Psychotherapeuten stattfinden. Hier spiegelt sich die unbefriedigende Situation wider, daß es noch keine gesetzliche Regelung für den Beruf des Psychologischen Psychotherapeuten gibt, die diesem die berufsrechtliche Gleichstellung mit dem Arzt und Heilpraktiker garantiert und ihn ebenfalls gleichberechtigt in das Leistungsrecht der gesetzlichen Krankenversicherung eingliedert. Hinsichtlich der Betonung der nachrangigen Stellung des Psychologischen Psychotherapeuten unterscheiden sich die Versicherungen beträchtlich - wenngleich dies in der Praxis kaum Bedeutung haben dürfte, da psychologische und ärztliche Kollegen in der Regel kooperativ miteinander umgehen.

Eine um sich greifende neuere Entwicklung sind Spartarife (z. B. Vereinte), in denen ambulante Psychotherapie aus dem Leistungskatalog ausgeschlossen ist. Solche Tarife sind aus unserer Sicht grundsätzlich abzulehnen. Sie gehen von der falschen - und im Einzelfall verhängnisvollen -

Voraussetzung aus, daß der einzelne Versicherte sein Risiko, psychisch zu erkranken. kalkulieren kann.

Eines zeigt unsere Übersicht über die private Versicherungslandschaft im Bereich Psychotherapie auch: Ware Psychotherapie in der Gesetzlichen Krankenversicherung nicht eine einheitlich 
definierte Regel-leistung, würde es ihr nicht anderes ergehen als in der PKV. Der freie Markt würde zu einer Zersplitterung und damit zu einem Qualitätsverlust in diesem Bereich führen. 

Verhaltenstherapie 1996;6;57-67 
Zum Zwecke einer sachgerechten Ausgestaltung und einer Vereinheitlichung des Leistungsbereiches Psycho logische Psychotherapie in der privaten Krankenversicherung hatten wir im April 1995 nach einem Gespräch mit dem Geschäftsführer des Verbandes der PKV Empfehlungen zu diesem Leistungsbereich vorgelegt. Wir müssen aber leider feststellen, daß die privaten Versicherungen bis jetzt kein Interesse gezeigt haben, sich mit diesen Problemen auseinanderzusetzen.

Die Broschüre bietet dem Therapeuten einen Überblick über das Erstattungsverhalten jeder einzelnen Ver-sicherung, sie enthält außerdem einen Abschnitt über die Beihilfe, die Postbetriebskrankenkasse B, die GOÄ und die Empfehlungen der Vereinigung zur Vereinheitlichung dieses Leistungsbereiches. Die Broschüre kann über die Geschäftsstelle bestellt werden. Der Preis lag bis zum

Redaktionsschluß noch nicht fest.

Geschäftsstelle der

Vereinigung der Kassenpsychotherapeuten:

Dipl.-Psych. Dieter Best

Riedsaumstraße 4a

67063 Ludwigshafen

Tel. (0621)637015

Fax (0621) 637016

Mitteilungen der Verbände 
Verhaltenstherapie 1996;6;57-67 
